УДК 621.396

\title{
СУММАРНАЯ СКОРОСТЬ БОЛЬШИХ МІМО-СИСТЕМ С ПОЛУОРТОГОНАЛЬНОЙ И ПРОИЗВОЛЬНОЙ ВЫБОРКОЙ ПОЛЬЗОВАТЕЛЕЙ В КАНАЛАХ С ВЫСОКИМ РАССЕИВАНИЕМ*
}

\author{
Т. А.ШЕЙХ, ДЖ. БОРА, А. ХУССЕЙН \\ Северо-восточный региональный институт науки и техники, \\ Индия, Нирджули, 791109, Аруначал-Прадеш
}

\begin{abstract}
Аннотация. Суммарная скорость передачи данных (sum-rate) больших MIMO-систем зависит от методик адекватного выбора пользователя и антенны. Наилучшие значения достигаются в случае, когда все выбранные пользователи взаимно ортогональны MOU (mutually orthogonal users), а антенны выбираются таким образом, чтоб получить максимальное усиление в канале. Если количество антенн большой МIMO-системы порядка сотни, вычислительная сложность и затраты подключения пользователя и выбора антенны очень высоки. Следовательно, для уменьшения сложности и затрат более предпочтительна полуортогональная выборка пользователей SUS (semi-orthogonal user selection). В каналах с релеевским затуханием может быть использована методика произвольной выборки пользователей RUS (random user selection), благодаря небольшим потерям в значении суммы скоростей по сравнению с методиками SUS и MOU. В данной работе рассмотрены алгоритмы подключения пользователей и выбора антенны, когда пользователи регистрировались с помощью полуортогональной меры, а выбор антенны осуществляется на основании максимального усиления в канале. Также представлен алгоритм подключения пользователя RUS и максимальном усилении в канале при выборе антенны. Исследованы характеристики общей скорости большой МІМО-системы с использованием указанных алгоритмов для случая релеевских каналов с высоким рассеиванием. Рассмотрены MMSE, ZFBF и MRT методики прекодирования, а также SNR. Исследовано влияние пространственного и пользовательского разнесения на суммарную скорость системы, использующей предложенные алгоритмы. Показано, что суммарная скорость, получаемая с помощью алгоритма-1 превышает скорость при использовании алгоритма-2 на 1-3\% при намного большей сложности.
\end{abstract}

Ключевые слова: большая МІМО-система; сумма скоростей; произвольная выборка пользователя; полуортогональная выборка пользователя; прекодирование

\section{1. ВВЕДЕНИЕ}

В настоящее время наиболее распространена беспроводная передача мультимедийных сообщений, которая требует высокой скорости передачи данных от пользователей и поставщиков услуг. Текущие разработки в области беспроводной связи преимущественно фокусируются на том, как увеличить скорость передачи данных пользователя при допустимых затратах.

Большие MIMO-системы (massive MIMO) имеют потенциальную возможность обеспечить высокую скорость передачи данных с

* Авторы выражают благодарность министерству электроники и информационных технологий и правительству Индии за финансовую поддержку данной работы и гранта № PhD-MLA-4 (96) / 2015-2016 от 05.11.2015. 


\section{БИБЛИОГРАФИЧЕСКИЙ СПИСОК}

1. Marzetta, T. L. "Noncooperative cellular wireless with unlimited numbers of base station antennas," IEEE Trans. Wireless Commun., Vol. 9, No. 11, p. 3590-3600, Nov. 2010. DOI: 10.1109/TWC.2010.092810.091092.

2. Sheikh, T. Ali; Bora, J.; Hussain, A. "A survey of antenna and user scheduling techniques for massive MIMO-5G wireless system," Proc. of Int. Conf. on Current Trends in Computer, Electrical, Electronics and Communication, 8-9 Sept. 2017, Mysore, India. IEEE, 2017, pp. 578-583. DOI: 10.1109/CTCEEC.2017.84551 77.

3. AL-Rawi, M.; AL-Rawi, M. "Performance of massive MIMO uplink system over Nakagami-m fading channel," Radioelectron. Commun. Syst., Vol. 60, No. 1, p. 13-17, 2017. DOI: $10.3103 / \mathrm{S} 0735272717010022$.

4. Liu, H.; Gao, H.; Yang, S.; Lv, T. "Low-complexity downlink user selection for massive MIMO systems," IEEE Syst. J., Vol. 11, No. 2, p. 1072-1083, Jun 2017. DOI: $10.1109 /$ JSYST.2015.2422475.

5. Mao, J.; Gao, J.; Liu, Y.; Xie, G. "Simplified semi-orthogonal user selection for MU-MIMO systems with ZFBF," IEEE Wireless Commun. Lett., Vol. 1, No. 1, p. 42-45, Feb. 2012. DOI: 10.1109/WCL.2012.010912.110 119.

6. Chia, S.; Gasparroni, M.; Brick, P. "The next challenge for cellular networks: Backhaul," IEEE Microw. Mag., Vol. 10, No. 5, p. 54-66, Aug. 2009. DOI: 10.1109/MMM.2009.932832.

7. Xu, G.; Liu, A.; Jiang, W.; Xiang, H.; Luo, W. "Joint user scheduling and antenna selection in distributed massive MIMO systems with limited backhaul capacity," China Commun., Vol. 11, No. 5, p. 17-30, May 2014. DOI: 10.1109/CC.2014.6880457.

8. Dong, Y.; Tang, Y.; Shenzhen, K. Zhang. "Improved joint antenna selection and user scheduling for massive MIMO systems," Proc. of 16th IEEE Int. Conf. on Computer and Information Science, 24-26 May 2017, Wuhan, China. IEEE, 2017, pp. 69-74. DOI: 10.1109/ ICIS.2017.7959971.

9. Gao, Y.; Jiang, W.; Kaiser, T. "Bidirectional branch and bound based antenna seletion in massive MIMO systems," Proc. of 26th Annual Int. Symp. on PIMRC, 30 Aug.-2 Sept. 2015, Hong Kong, China. IEEE, 2015, pp. 563-568. DOI: 10.1109/PIMRC.2015.7343363.

10. Benmimoune, M.; Driouch, E.; Ajib, W.; Massicotte, D. "Joint transmit antenna selection and user scheduling for massive MIMO systems," Proc. of IEEE Wireless Communication and Networking Conf., 9-12 Mar. 2015, New Orleans, LA, USA. IEEE, 2015, pp. 381-386. DOI: 10.1109/WCNC.2015.7127500. 